\title{
Association between 5-lipoxygenase expression, and malignant behaviors and poor prognosis in esophageal squamous cell carcinoma
}

\author{
CHUN-YING BAI ${ }^{1,2 *}$, JUN-YI ZHANG ${ }^{1,3^{*}}$, TIE-WEI SHI ${ }^{2}$, YU-QIN BAI $^{3}$, \\ BING-LI WU ${ }^{1,4}$, ZE-PENG DU ${ }^{5}$, ZHI-YONG WU ${ }^{1,6}$, XIU-E XU ${ }^{1,7}$, SHAO-HONG WANG $^{5}$, \\ JIAN-YI WU ${ }^{1,5}$, RUI-YUN TE ${ }^{2}$, JING-YI ZHANG ${ }^{3}$, LI-YAN XU ${ }^{1,7}$ and EN-MIN LI ${ }^{1,4}$
}

\begin{abstract}
${ }^{1}$ The Key Laboratory of Molecular Biology for High Cancer Incidence Coastal Chaoshan Area, Shantou University, Medical College, Shantou, Guangdong 515041; ${ }^{2}$ Research Center of Molecular Medicine; ${ }^{3}$ Department of Pathology, Medical College of Chifeng University, Chifeng, Inner Mongolia 024000; ${ }^{4}$ Department of Biochemistry and Molecular Biology, Shantou University Medical College; Departments of ${ }^{5}$ Pathology and ${ }^{6}$ Oncology Surgery, Shantou Central Hospital, Affiliated Shantou Hospital of Sun Yat-sen University; ${ }^{7}$ Institute of Cancer Pathology,

Shantou University Medical College, Shantou, Guangdong 515041, P.R. China
\end{abstract}

Received July 6, 2016; Accepted March 14, 2018

DOI: $10.3892 / \mathrm{ol} .2018 .8527$

\begin{abstract}
LO) catalyzes the first step of arachidonic acid metabolism to inflammatory mediator leukotrienes. The present study assessed 5-LO expression in esophageal squamous cell carcinoma (ESCC) tissue specimens for associations with clinicopathological and survival data from patients, then explored 5-LO activity in ESCC cells in vitro. 5-LO expression was detected in tissue microarrays containing 297 ESCC samples using immunohistochemistry. Kaplan-Meier curves were used to analyze the survival significance of 5-LO expression and relative risk was evaluated using the multivariate Cox proportional hazards model. Cultured tumor cells were subjected to gene transfection, western blotting, and cell migration and proliferation assays. 5 -LO protein was primarily expressed in normal cell cytoplasm and/or membrane, and never in the whole cytoplasm, whereas 5-LO was expressed diffusely in ESCC tissues with nearly homogeneous whole-cytoplasm staining. 5-LO
\end{abstract}

Correspondence to: Dr En-Min Li, Department of Biochemistry and Molecular Biology, Shantou University Medical College, 22 Xinling Road, Shantou, Guangdong 515041, P.R. China

E-mail:nmli@stu.edu.cn

Dr Li-Yan Xu, Institute of Cancer Pathology, Shantou University Medical College, 22 Xinling Road, Shantou, Guangdong 515041, P.R. China

E-mail: lyxu@stu.edu.cn

*Contributed equally

Key words: esophageal squamous cell carcinoma, 5-lipoxygenase, prognosis, biomarker, tumor cell malignant behavior expression was significantly associated with tumor regional lymph node metastasis $(\mathrm{P}=0.013)$ and $\mathrm{pTNM}$ stage $(\mathrm{P}=0.004)$. 5 -LO expression was associated with poor overall survival $(\mathrm{P}=0.029)$. Multivariate analysis demonstrated that 5-LO overexpression was an independent prognostic factor for ESCC patients ( $\mathrm{P}=0.041)$. Furthermore, the inhibition of 5-LO expression reduced ESCC cell viability and migration in vitro. These data provide further evidence that the upregulation of 5-LO expression is associated with advanced stages of disease and poor ESCC prognosis, and that 5-LO expression may independently predict overall survival in patients with ESCC. The inhibition of 5-LO expression reduced ESCC malignant behavior in vitro.

\section{Introduction}

Esophageal cancer is a common type of malignancy worldwide with an estimated 482,300 new cases and 406,800 mortalities globally in 2008 (1). In China, esophageal cancer is also recognized as a high-incidence tumor and greatly contributes to global esophageal cancer incidence (2). Histologically, the majority of patients with esophageal cancer have esophageal squamous cell carcinoma (ESCC) worldwide and in China, whereas esophageal adenocarcinoma may occur more frequently in developed countries, including the USA (3). The primary worldwide risk factors of ESCC include tobacco smoking and alcohol consumption; in China, the consumption of salted vegetables or other nutritional factors are also associated with a high incidence of ESCC (3). To date, the exact ESCC pathogenesis and mechanisms remain unclear; a deeper understanding of ESCC development and progression may lead to the identification of novel therapeutic strategies for the regulation of this fatal disease.

5-lipoxygenase (5-LO) is an important enzyme that catalyzes arachidonic acid biosynthesis to hydroxyeicosatetraenoic 
acids or leukotrienes (LTs). Previous studies demonstrated that 5-LO may act through a number of signaling pathways to promote tumorigenesis, and 5-LO expression was identified to be upregulated in several human cancer types, including breast (4), lung (5), pancreatic (6), colon (7), prostate $(8,9)$ and esophageal cancer (10-12). Two previous studies on esophageal cancer also indicated that the inhibition of 5-LO enzymatic activity was able to induce apoptosis in tumor cells $(13,14)$. However, data is currently lacking regarding whether upregulated 5-LO expression is associated with the clinical importance and prognosis of ESCC. Thus, the aims of the present study were to investigate 5-LO expression in a large ESCC cohort for its association with clinicopathological and survival data, and to then explore 5-LO expression or knockdown in the regulation of ESCC proliferation and migration capacity in vitro. The results of the present study may provide further insights into the role of 5-LO in ESCC progression and may support the future targeting of 5-LO in the management or treatment of patients with ESCC.

\section{Materials and methods}

Patients. A total of 297 tissue samples were collected from patients with ESCC who underwent surgical treatment at Shantou City Center Hospital (Shantou, China) between January 2007 and December 2011. All patients were pathologically diagnosed and confirmed with ESCC in the Anatomic Pathology Department of the Hospital according to the 8th edition of the tumor-node-metastasis (TNM) classification released by the International Union against Cancer and guidelines of the World Health Organization Pathological Classification of Tumors (15). Among the patients, there were 232 males and 65 females with a medium age of 59 years (range, 39-88 years). Patients underwent surgical tumor resection and received radiochemotherapy thereafter. All patients were followed up regularly until they succumbed. The maximum length of follow-up was 60 months with a medium of 26.77 months. Clinicopathological data was also collected from the medical records, including gender, age, stage of disease and tumor differentiation. The study was performed in accordance with the Declaration of Helsinki and Good Clinical Practice guidelines. The present study was approved by the Ethics Committee of the Shantou City Center Hospital and all enrolled patients provided written informed consent.

Tissue samples and construction of tissue microarrays. Paraffin blocks from the 297 tissue specimens were retrieved from the Pathology Department, and the tissue sections were prepared for hematoxylin and eosin (HE) staining to confirm the diagnosis and to select areas for the construction of tissue microarrays as previously described (16). Briefly, representative areas of tumor tissues were selected from paraffin blocks after reviewing the HE-stained tissue sections. From each tissue specimen, two tissue cores were selected with a diameter of $1.8 \mathrm{~mm}$ and length of 1.0-3.0 mm, which depended on the depth of the tissue specimens in the donor blocks. A new paraffin block was then precisely generated with the arrayed cores from the original paraffin blocks. The quality of tissue samplings was verified via paraffin-embedded tissue sectioning and HE staining. The tissue microarray sections were then baked at $56^{\circ} \mathrm{C}$ overnight and subjected to immunohistochemistry.

Immunohistochemistry. For immunohistochemistry, 10\% neutral buffered formalin-fixed, paraffin-embedded specimens were cut into $4-\mu$ m-thick sections. The sections were dewaxed in xylene and rehydrated in a series of graded alcohols. Subsequently, slides were submerged in a peroxidase quenching solution containing 30\% hydrogen peroxide and absolute methanol at a ratio of 1:9, respectively, for $10 \mathrm{~min}$. Subsequent to rinsing in phosphate-buffered saline, antigen retrieval from the tissue was performed by autoclaving in $0.01 \mathrm{~mol} / \mathrm{l}$ sodium citrate buffer $(\mathrm{pH} 6.0)$ at $120^{\circ} \mathrm{C}$ for $3 \mathrm{~min}$. Next, sections were blocked in $10 \%$ normal goat serum (ZSGB-BIO; OriGene Technologies, Beijing, China) for $10 \mathrm{~min}$ at room temperature, and then incubated overnight at $4^{\circ} \mathrm{C}$ with rabbit monoclonal antibody against 5-LO (1:100 dilution; AP7856C, San Diego, CA, USA). Then, the sections were subjected to immunostaining using the SuperPicture Polymer Detection kit (Product code 879363, Thermo Fisher Scientific, Inc., Waltham, USA), which contained a ready-to-use secondary antibody. The slides were examined with an Olympus IX51 light microscope (Olympus, Tokyo, Japan) at magnification, $\mathrm{x} 100$. The immunostained tissue microarray section was then reviewed and independently evaluated by two histopathologists who did not know the clinicopathological data of the patients. Immunoreactivity was evident according to brown color on the cell membrane or in the cytoplasm. In the study, the 5-LO expression level and the subcellular 5-LO distribution were respectively recorded. Regarding the 5-LO expression level, the scores that reflected the staining intensity were as follows: 3 , strong; 2 , moderate; 1 , weak; and 0 , negative, and the staining percentages were ranked as $4,>75 ; 3$, $51-75 ; 2,26-50 ; 1,5-25$; and $0,<5 \%$. Final scores were recorded by multiplying the intensity and percentage scores to produce a 0 to 12 digit scoring. For statistical analyses, scores of 0 to 4 were regarded as low expression, whereas 5-12 indicated high expression.

Cell lines and culture. The human ESCC cell lines SHEEC, KYSE510, KYSE150, KYSE180, and KYSE450 were obtained from the Key Laboratory of Molecular Biology for High Cancer Incidence Coastal Chaoshan Area, Shantou University Medical College (Shantou, China) and cultured in RPMI-1640 or Dulbecco's modified Eagle's medium/F-12 medium containing $10 \%$ fetal calf serum (Thermo Fisher Scientific, Inc.) at $37^{\circ} \mathrm{C}$ in a humidified $5 \% \mathrm{CO}_{2}$ atmosphere. It is necessary to emphasize that the SHEEC cell line was developed and provided by the Department of Tumor Pathology, Medical College of Shantou University (17).

Protein extraction and western blotting. Total cellular protein was extracted from esophageal cancer cell lines using RIPA buffer (Maygene, Guangzhou, China). Protein determination was performed using the BCA method, and $80 \mu \mathrm{g}$ total protein samples were separated using 10\% SDS-PAGE and transferred on to a polyvinylidene difluoride membrane (EMD Millipore, Billerica, MA, USA). The membrane was then blocked in 5\% skim milk at the room temperature for $1 \mathrm{~h}$ and then incubated at $4^{\circ} \mathrm{C}$ overnight with an anti-5-LO antibody (cat. no., 3289, 
Cell Signaling Technology, Inc., Danvers, MA, USA) at a dilution of 1:500 or with an anti- $\beta$-actin antibody (cat. no., A2066; Sigma-Aldrich; Merck KGaA, Darmstadt, Germany) at a dilution of 1:1,000. On the next day, the membrane was washed with PBS-Tween 20 thrice and further incubated with horseradish peroxidase-conjugated secondary antibody (cat. no., sc-2004; Santa Cruz Biotechnology, Inc., Dallas, TX, USA) at a dilution of 1:5,000 at room temperature for $2 \mathrm{~h}$. The protein bands were subsequently visualized with ECL reagent (Thermo Fisher Scientific, Inc.) and the FluorChem 8900 image analysis system (ProteinSimple, San Jose, CA, USA) was used to capture images and quantitatively analyze 5-LO levels following normalization to the level of $\beta$-actin.

Vector construction and gene transfection. Human recombinant 5-LO eukaryotic expression plasmid vector pcDNA3.1 (+)/5-LO was gifted by Dr Eiko Matsui from Gifu University (Gifu, Japan). A total of $50 \mu \mathrm{g}$ 5-LO cDNA-containing plasmid was transfected into KYSE 150 cells, and an empty plasmid was used as a negative control using Lipofectamine ${ }^{\circledR} 2000$ transfection reagent (Invitrogen; Thermo Fisher Scientific, Inc.) according to the manufacturer's instructions. Furthermore, two different 5-LO small interfering (si)RNA (150 pmol) sequences $(18,19)$ were also designed to knock down 5-LO expression in ESCC cells. These sequences were synthesized by GenePharma (Shanghai, China): siRNA-1, 5'-AUUGCC CUGAAAAACUGUG-3' and siRNA-2, 5'-AGAAAUCCC AAGAUCAGUG-3'. These oligonucleotides were transiently transfected into ESCC SHEEC cells using Lipofectamine 2000 transfection reagent according to the manufacturer's protocol and the nonsense siRNA (5'-UUCUCCGAACGU GUCACGUTT-3') was transfected into the cells as a negative control.

Tumor cell proliferation and migration assays. A real-time cell electronic sensing RTCA DP analyzer with Galaxy 48R (Real-Time Cell analyzer, Roche Applied Science, Penzberg, Germany) was used to assess the effects of 5-LO manipulation on the regulation of ESCC cell viability and migration capacity. Briefly, human ESCC cells transfected with 5-LO cDNA or siRNA were seeded in E-plate to assess changes in cell proliferation or the cell invasion and migration (CIM)-plate to assess changes in cell migration followed by culturing for up to 2 days. Using the cell index (CI), data was obtained on changes regarding the cell proliferation and the migration rates. A total of 10,000 cells in RPMI-1640 medium containing $10 \%$ bovine serum albumin (BSA; Thermo Fisher Scientific, Inc.) were added to each well of the E-plate. In addition, a total of 50,000 cells in DMEM/F-12 medium containing 10\% BSA were added to each well of the CIM-plate.

Statistical analysis. Associations between 5-LO expression and clinicopathological characteristics, including gender, age, tumor TNM classification, histology, size, lymph node metastasis and distant metastasis, were evaluated using Pearson's chi-square test. A log-rank test was applied to analyze the Kaplan-Meier curves for associations between 5-LO expression and overall survival (OS). OS was defined as the duration between surgery and mortality associated with esophageal cancer. Associations between the clinicopathological characteristics and 5-LO expression with overall survival were assessed using the multivariate Cox proportional hazards regression model. Data are represented as the mean \pm standard deviation. All analyses were performed with SPSS for Windows 13.0 software (SPSS, Inc., Chicago, IL, USA). All $\mathrm{P}$-values were two-tailed and $\mathrm{P} \leq 0.05$ was considered to indicate a statistically significant difference.

\section{Results}

Differential 5-LO expression in ESCC and pericarcinoma esophageal tissues. 5-LO expression was detected in 297 ESCC samples and 66 pericarcinoma esophageal tissues using immunohistochemical staining (Fig. 1). Specifically, in pericarcinoma esophageal tissues, 5-LO was primarily expressed in the cell cytoplasm or membrane (Fig. 1A and B). In ESCC, 5-LO expression was localized in the cytoplasm and/or nucleus of the tumor cells, but the expression intensity and the percentage of positive cells were upregulated (Fig. 1C-G). 5 -LO staining patterns in cancer cells were heterogeneous and high 5-LO expression (sum index score $\geq 5$ ) was detected in $122 / 297$ (41.1\%) tumor samples, but only 6/66 (11\%) pericarcinoma esophageal tissues $(\mathrm{P}<0.05)$.

Association between 5-LO expression and clinicopathological data. Statistical analysis revealed an association between 5-LO expression and regional lymph node metastasis (48.3 vs. $\left.34 \% ; \mathrm{X}^{2}=0.145 ; \mathrm{P}=0.013\right)$ and advanced pathological (p)TNM stage $\left(50.4\right.$ vs. $\left.33.5 \% ; \mathrm{X}^{2}=0.17 ; \mathrm{P}=0.004\right)$. However, no significant associations were identified between 5-LO expression and other clinicopathological factors, including gender, age, tumor location or distant metastasis (Table I).

Association between 5-LO expression and overall survival in ESCC patients. The median post-operative survival time was 26.77 months. A total of $122(41.1 \%)$ patients had ESCC expressing high 5-LO and 175 (58.9\%) patients had ESCC expressing low 5-LO. The Kaplan-Meier curves showed that stronger 5-LO immunoreactivity was significantly associated with poorer overall survival in patients with ESCC ( $\mathrm{P}=0.029$; Fig. 2).

Using a Cox proportional hazards model, a multivariate analysis was performed to assess predictors for overall survival of this cohort of patients. It was demonstrated that pTNM stage $(\mathrm{P}<0.001)$, depth of tumor invasion $(\mathrm{P}=0.031)$, Histology $(\mathrm{P}=0.012)$, age $(\mathrm{P}=0.035)$, regional lymph node metastasis $(\mathrm{P}<0.001)$ and 5-LO expression $(\mathrm{P}=0.041)$ were independent prognostic factors for survival in patients with ESCC (Table II).

5-LO expression regulates ESCC cell viability and migration in vitro. The expression of 5-LO in ESCC cell lines was analyzed and manipulated to determine the effects on cell proliferation, and migration (Fig. 3). It was revealed that SHEEC cells had a high level of 5-LO protein expression, KYSE510 exhibited intermediate levels of 5-LO expression, and KYSE150, KYSE180 and KYSE450 had low expression levels (Fig. 3A). Next, 5-LO expression was manipulated in the selected ESCC cell lines via transfection with 5-LO cDNA or siRNA. Two constructs of 5-LO siRNAs 

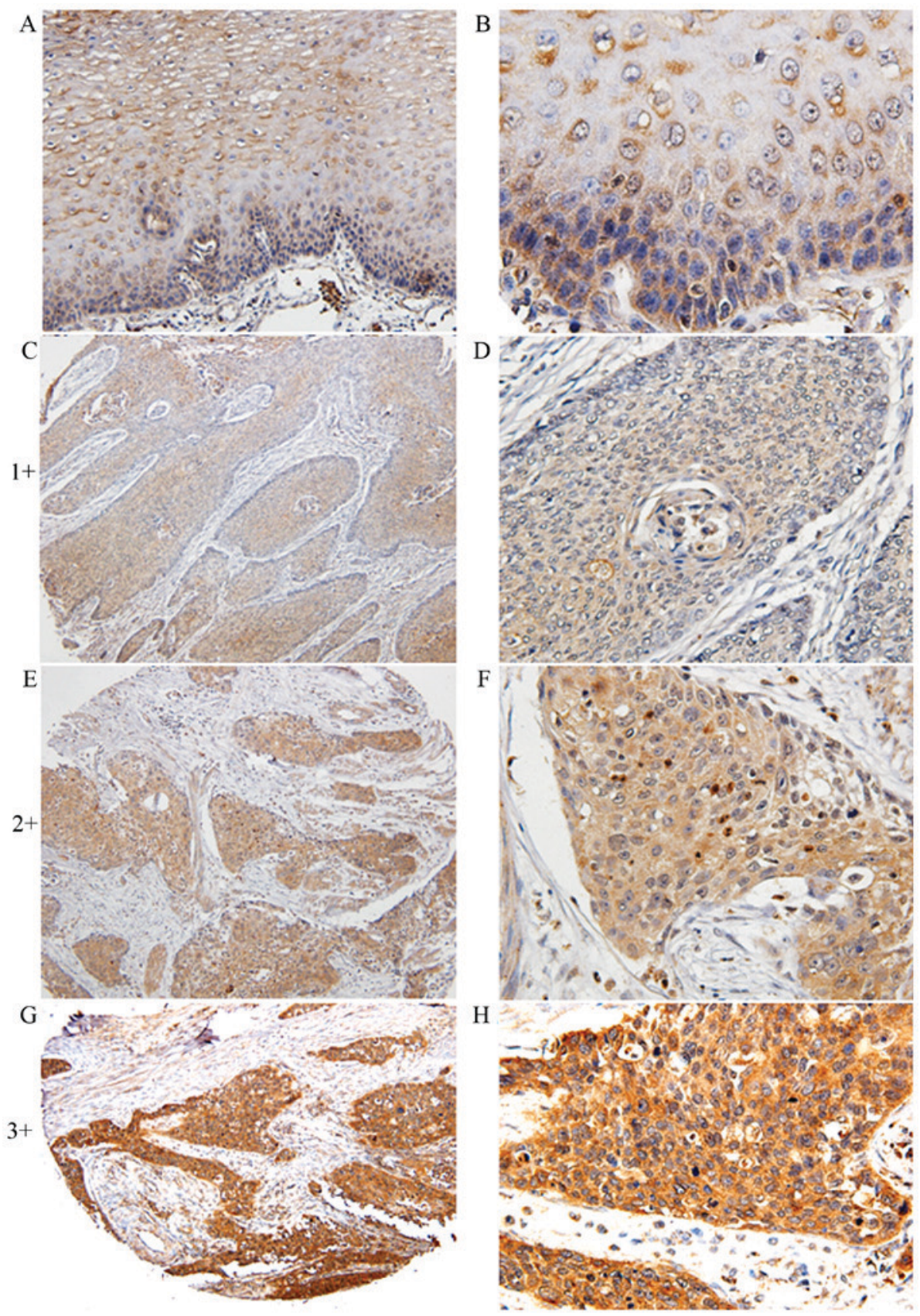

Figure 1. Differential expression of 5-LO expression in normal esophageal epithelia and ESCC tissues detected via immunohistochemistry. (A and B), 5-LO expression in normal esophageal epithelium. (C and D), weak staining (1+) of 5-LO protein in ESCC. (E and F), moderate staining (2+) of 5-LO protein in ESCC. (G and H), strong staining (3+) of 5-LO protein in ESCC. Original magnification, x100 and x400. ESCC, esophageal squamous cell carcinoma; 5-LO, 5-lipoxygenase.

were transfected into SHEEC cells that expressed high levels of 5-LO protein, and these two siRNAs were able to knock down 5-LO expression (Fig. 3B). Real-time analysis of cell proliferation and migration revealed that 5-LO knockdown reduced tumor cell proliferation and migration capacities compared with the control (Fig. 3D and E). Regardless of cell proliferation or mobile ability, for $0-45 \mathrm{~h}$ after siRNA interference, the level of 5-LO expression relative to the control group was significantly decreased, particularly in the 5-LO siRNA-1-transfeced cells.

Furthermore, plasmids carrying 5-LO cDNA were transfected into KYSE150 cells, which increased the protein expression of 5-LO (Fig. 3C). Real-time analysis of cell proliferation demonstrated that 5-LO significantly increased cell proliferation for 0-63 h after 5-LO transfection compared with the vector control-transfected cells (t-test, $\mathrm{P}<0.05$, Fig. $3 \mathrm{~F}$, the statistical significance on the relevant graphs using *,); however, the migration of ESCC cells was not significantly affected by 5 -LO expression for $0-70 \mathrm{~h}$ ( $\mathrm{P}>0.05$; Fig. 3G).

\section{Discussion}

5-LO was originally reported to be primarily expressed in leukocytes, including B-lymphocytes, polymorphonuclear leukocytes (neutrophils and eosinophils), mast cells, monocytes/macrophages and foam cells or dendritic cells of human 
Table I. Association between 5-LO expression and parameters in with esophageal squamous cell carcinoma.

\begin{tabular}{|c|c|c|c|c|}
\hline Clinical parameters & Low 5-LO expression & High 5-LO expression & $X^{2}$ & P-value \\
\hline Age (years) & & & 0.008 & 0.907 \\
\hline$<59$ & 89 & 63 & & \\
\hline$\geq 59$ & 86 & 56 & & \\
\hline Sex & & & 0.055 & 0.393 \\
\hline Male & 140 & 92 & & \\
\hline Female & 35 & 30 & & \\
\hline Tumor size (cm) & & & 0.073 & 0.216 \\
\hline$\leq 3$ & 37 & 28 & & \\
\hline $3-5$ & 77 & 63 & & \\
\hline$>5$ & 59 & 31 & & \\
\hline Tumor location & & & 0.021 & 0.735 \\
\hline Upper & 11 & 8 & & \\
\hline Middle & 77 & 56 & & \\
\hline Lower & 87 & 58 & & \\
\hline Differentiation grade & & & 0.088 & 0.119 \\
\hline $\mathrm{G} 1$ & 32 & 13 & & \\
\hline G2 & 129 & 98 & & \\
\hline G3 & 14 & 11 & & \\
\hline Invasive depth & & & 0.094 & 0.134 \\
\hline $\mathrm{T} 1+\mathrm{T} 2$ & 39 & 18 & & \\
\hline $\mathrm{T} 3+\mathrm{T} 4$ & 136 & 104 & & \\
\hline Regional lymph nodes & & & 0.145 & $0.013^{\mathrm{a}}$ \\
\hline N0 & 99 & 51 & & \\
\hline $\mathrm{N} 1+\mathrm{N} 2+\mathrm{N} 3$ & 76 & 71 & & \\
\hline pTNM stage & & & 0.17 & $0.004^{\mathrm{a}}$ \\
\hline $\mathrm{I}+\mathrm{II}$ & 109 & 55 & & \\
\hline III+IV & 66 & 67 & & \\
\hline
\end{tabular}

${ }^{\mathrm{a}} \mathrm{P}<0.05$. pTNM, pathological tumor-node-metastasis; 5-LO, 5-lipoxygenase.

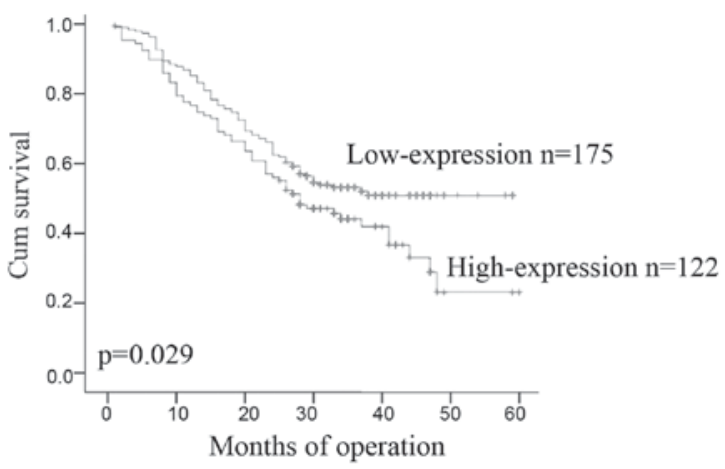

Figure 2. Association of 5-LO expression with overall survival in 297 ESCC patients. Kaplan-Meier curve analysis of overall survival in 297 patients stratified according to 5-LO expression. Patients with ESCC expressing low 5-LO had longer survival than those with ESCC expressing high 5-LO $(\mathrm{P}=0.029)$. ESCC, esophageal squamous cell carcinoma; 5-LO, 5-lipoxygenase.

atherosclerotic tissue (20). The function of 5-LO is to convert arachidonic acid to hydroxyeicosatetraenoic acids (5-HETE) or LTs. However, previous studies revealed that an increased expression of 5-LO was associated with human carcinogenesis (4-12). In esophageal cancer, a previous cDNA microarray study of 14,803 genes identified nine genes that were upregulated and 36 genes that were downregulated in ESCC tissue specimens (11). In addition, nine of the altered gene expressions were associated with arachidonic acid metabolism and 5-LO was upregulated in esophageal cancer (11). The present study, together with other studies $(10,12)$, indicated that 5-LO upregulation may play a role in ESCC development; thus, the current study aimed to further investigate 5-LO expression in ESCC tissue specimens, and its association with clinicopathological and survival data from patients with ESCC. The data of the current study revealed that 5-LO was highly expressed in ESCC tissue specimens, expression of which was significantly associated with advanced stage of disease and tumor lymph node metastasis, and was identified as an independent predictor for overall survival of patients. Additionally, the limited in vitro data indicated that 5-LO expression induced tumor cell proliferation, whereas the knockdown of 5-LO expression suppressed tumor cell proliferation and migration. The current data indicated that 5-LO expression may serve as 
Table II. Multivariate Cox regression analysis of clinicopathological factors for risk prediction in 297 patients with esophageal squamous cell carcinoma.

\begin{tabular}{lccr}
\hline Factors & Hazard ratio & 95\% Confidence interval & P-value \\
\hline Age & 1.419 & $1.026-1.964$ & 0.035 \\
Gender & 0.912 & $0.618-1.346$ & 1.346 \\
Tumor size & 0.844 & $0.583-1.222$ & 0.369 \\
Tumor location & 1.311 & $0.713-2.41$ & 0.383 \\
Invasive depth & 0.482 & $0.248-0.934$ & 0.031 \\
Histology & 0.433 & $0.226-0.831$ & 0.012 \\
Lymph node metastasis & 1.992 & $1.429-2.779$ & $<0.001$ \\
pTNM stage & 2 & $1.44-2.778$ & $<0.001$ \\
5-LO high expression & 1.412 & $1.014-1.967$ & 0.041 \\
\hline
\end{tabular}

pTNM, pathological tumor-node-metastasis; 5-LO, 5-lipoxygenase.

A

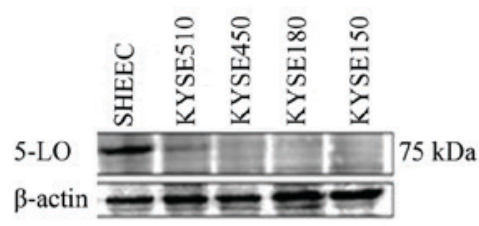

B

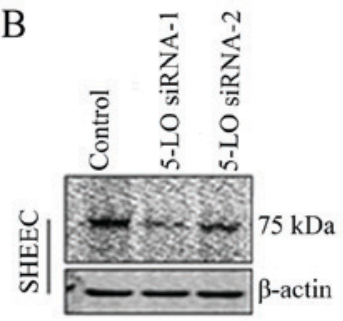

$\mathrm{C}$

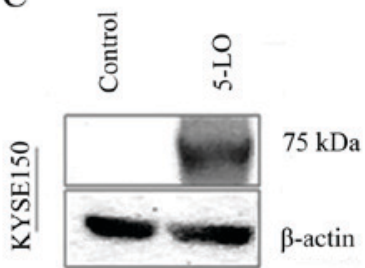

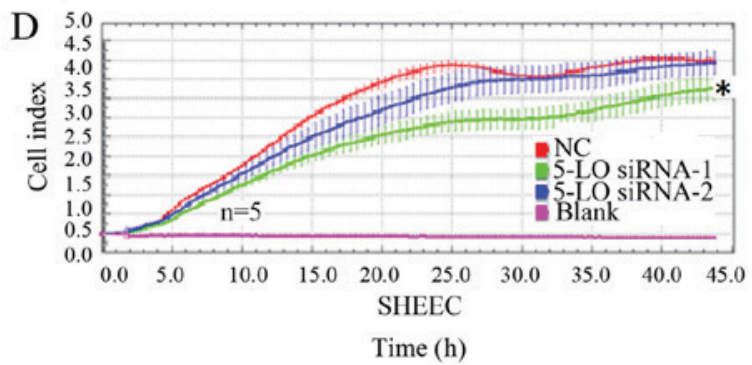

Time (h)

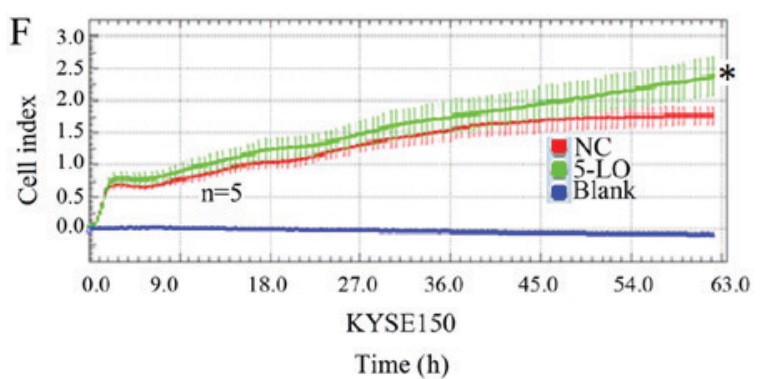

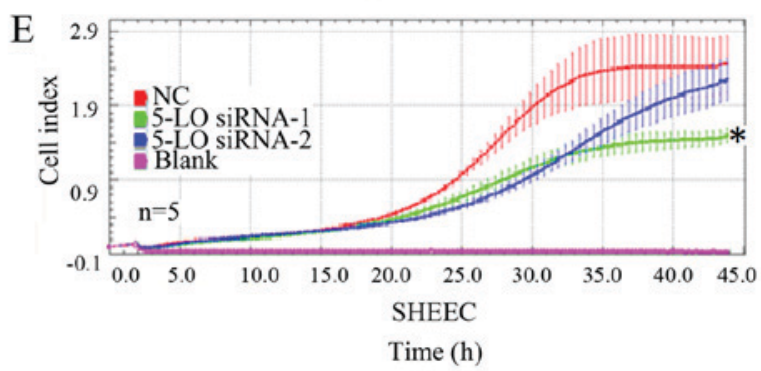

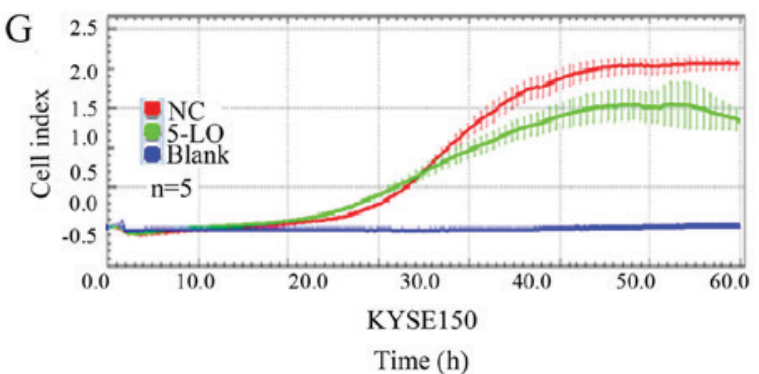

Figure 3. Effects of 5-LO knockdown or expression on the regulation of tumor cell proliferation and migration in vitro. The statistical significance on the relevant graphs using *. (A) Western blot analysis. 5-LO expression was assessed using Western blot in various ESCC cell lines. (B) Western blot analysis. ESCC SHEEC cells with highly expressed 5-LO were transfected with two different constructs of 5-LO siRNA and then subjected to Western blot analysis for 5-LO expression. (C) Western blot analysis. ESCC KYSE150 cells with low 5-LO expression were transfected with 5-LO cDNA and then subjected to western blot analysis for 5-LO expression. (D) Cell proliferation assay. 5-LO-knocked down SHEEC cells were grown and subjected to cell proliferation assay. 5-LO knockdown significantly inhibited tumor cell proliferation ( $\mathrm{P}<0.05)$, especially in the 5-LO siRNA-1 group. (E) Tumor cell migration assay. The duplicated cells were subjected to tumor cell migration assay. 5-LO knockdown significantly inhibited tumor cell migration (P<0.05), especially in the 5-LO siRNA-1 group. (F) and (G) Cell proliferation and migration assays. 5-LO cDNA-transfected KYSE150 cells were grown and subjected to cell proliferation or migration assay, respectively. 5-LO expression significantly promoted cell proliferation $(\mathrm{F}, \mathrm{P}<0.05)$ but did not impact tumor cell migration $(\mathrm{G}, \mathrm{P}>0.05)$. ESCC, esophageal squamous cell carcinoma; 5-LO, 5-lipoxygenase; siRNA, small interferring RNA.

a prognostic marker and that targeting 5-LO may represent a viable therapeutic strategy in patients with ESCC.
Numerous studies have verified that 5-LO is overexpressed in a variety of tumors. Increased 5-LO expression 
was observed in glioblastoma cell lines and astrocytoma tissue specimens (21). The current study demonstrated that 5-LO expression was upregulated in ESCC tissues compared with pericarcinoma tissue, and that high 5-LO expression was associated with ESCC metastasis to the regional lymph nodes and advanced pTNM stage. The results of the present study are consistent with previous studieson other cancer types (4-9); for example, 5-LO expression was associated with lymph node metastasis in breast cancer (4). To the best of our knowledge, the current study was the first to associate 5-LO expression with poor overall survival in patients with ESCC. This is consistent with previous studies on other cancer types demonstrating an association between 5-LO expression and poor survival in patients with high-grade astrocytoma, and breast cancer $(4,20)$.

Furthermore, a larger panel of data supported that the 5-LO signaling pathway exerts profound effects on human tumorigenesis and progression, and that targeting 5-LO may lead to cancer treatment or prevention (20-25). However, the mechanism underlying how 5-LO promotes malignant transformation and malignant tumor behavior in vivo remains unclear. The present study revealed that the knockdown of 5-LO expression inhibited ESCC proliferation and migration capacity, whereas 5-LO overexpression promoted ESCC cell proliferation, but did not promote tumor cell migration. The current in vitro data are preliminary, and further studies are required to investigate the effects of 5-LO knockdown and expression in the regulation of ESCC viability, apoptosis, migration, invasion, and independent growth in soft agar, as well as the underlying molecular mechanism. In other cancer types, 5-LO was revealed to enhance cell proliferation and inhibit apoptosis (22). 5-LO was also reported to selectively inhibit tumor suppressor gene p53 transcription (23). Furthermore, 5-LO expression promoted tumor angiogenesis by inducing the expression of vascular endothelial growth factor (VEGF) (24), which is currently one of the strongest targets in promoting or generating tumor blood vessel formation (20). 5-LO was reported to induce VEGF transcription through 5-HETE, a product of 5-LO (20), whereas 5-LO inhibitors were able to decrease VEGF expression in colon cancer cells (25). These data clearly demonstrated that targeting 5-LO expression or activity may modulate certain human cancer activity; however, the arachidonic acid metabolism pathway is complicated and difficult to regulate. Thus, further studies are warranted to determine whether targeting 5-LO is sufficient for regulating ESCC.

In conclusion, the results of the current study revealed that increased 5-LO expression in ESCC tissue specimens is associated with ESCC metastasis to the lymph node and advanced stages of disease. Increased 5-LO expression was also identified as an independent predictor for ESCC survival. Furthermore, the inhibition of 5-LO expression suppressed the proliferation and migration of ESCC cells in vitro. These results supported that $5-\mathrm{LO}$ is a potential target for the prevention and treatment of esophageal cancer in clinical.

\section{Acknowledgements}

The authors would like to thank the Inner Mongolia Key Laboratory of Human Genetic Diseases (Medical College of Chifeng University, Chifeng, Inner Mongolia, China), for provide cell culture instruments for this study.

\section{Funding}

The present study was supported in part by the Natural Science Foundation of China (grant no. 81360331 to CB, grant no. 81201844 to JZ and grant no. 81502138 to ZD), the National Basic Research Program (grant no. 2012CB526600 to LX), the National High Technology Research and Development Program of China (grant no. 2012AA02A503 to EL) and the Natural Science Foundation of China-Guangdong Joint Fund (grant no. U1301227 to LX).

\section{Availability of data and materials}

The datasets used and/or analyzed during the current study are available from the corresponding author on reasonable request

\section{Authors' contributions}

EML and LYX designed the study. CYB and BLW were responsible for statistical analysis. TWS and CYB were responsible for cell culture, protein extraction and western blotting. ZPD, ZYW and JYW collected tissue samples and clinical data of patients. YQB and JuYZ were responsible for tumor cell proliferation and migration assays. SHW and RYT were responsible for collection of follow-up data for ESCC patients after surgery. XEX was responsible for construction of tissue microarrays. The immunostained tissue microarray section was reviewed and evaluated by YQB and JiYZ. CYB was a major contributor in writing the manuscript. EML, LYX and JiYZ revised the article. All authors read and approved the final manuscript.

\section{Ethics approval and consent to participate}

This study was approved by the Ethics Committee of Shantou University Medical College. Written informed consents were obtained from all the study participants.

\section{Consent for publication}

All patients provided written informed consent for the publication of their data.

\section{Competing interests}

The authors declare that they have no competing interests.

\section{References}

1. Jemal A, Bray F, Center MM, Ferlay J, Ward E and Forman D: Global cancer statistics. CA Cancer J Clin 61: 69-90, 2011.

2. Chen W: Cancer statistics: Updated cancer burden in China. Chinese J Cancer Res 27: 1, 2015.

3. Tran GD, Sun XD, Abnet CC, Fan JH, Dawsey SM, Dong ZW, Mark SD, Qiao YL and Taylor PR: Prospective study of risk factors for esophageal and gastric cancers in the Linxian general population trial cohort in China. Int J Cancer 113: 456-463, 2005.

4. Jiang WG, Douglas-Jones AG and Mansel RE: Aberrant expression of 5-lipoxygenase-activating protein (5-LOXAP) has prognostic and survival significance in patients with breast cancer. Prostaglandins Leukot Essent Fatty Acids 74: 125-134, 2006. 
5. Ou D, Bonomi P, Jao W, Jadko S, Harris JE and Anderson KM: The mode of cell death in $\mathrm{H}-358$ lung cancer cells cultured with inhibitors of 5-lipoxygenase or the free radical spin trap, NTBN. Cancer Lett 166: 223-231, 2001.

6. Hennig R, Ding XZ, Tong WG, Schneider MB, Standop J, Friess H, Büchler MW, Pour PM and Adrian TE: 5-Lipoxygenase and leukotriene $\mathrm{B}(4)$ receptor are expressed in human pancreatic cancers but not in pancreatic ducts in normal tissue. Am J Pathol 161: 421-428, 2002.

7. Ihara A, Wada K, Yoneda M, Fujisawa N, Takahashi $\mathrm{H}$ and Nakajima A: Blockade of leukotriene B4 signaling pathway induces apoptosis and suppresses cell proliferation in colon cancer. J Pharmacol Sci 103: 24-32, 2007.

8. Matsuyama M, Yoshimura R, Mitsuhashi M, Hase T, Tsuchida K, Takemoto Y, Kawahito Y, Sano H and Nakatani T: Expression of lipoxygenase in human prostate cancer and growth reduction by its inhibitors. Int J Oncol 24: 821-827, 2004.

9. Gupta S, Srivastava M, Ahmad N, Sakamoto K, Bostwick DG and Mukhtar H: Lipoxygenase-5 is overexpressed in prostate adenocarcinoma. Cancer 91: 737-743, 2001.

10. Hoque A, Lippman SM, Wu TT, Xu Y, Liang ZD, Swisher S, Zhang H, Cao L, Ajani JA and Xu XC: Increased 5-lipoxygenase expression and induction of apoptosis by its inhibitors in esophageal cancer: A potential target for prevention. Carcinogenesis 26 785-791, 2005

11. Zhi H, Zhang J, Hu G, Lu J, Wang X, Zhou C, Wu M and Liu Z: The deregulation of arachidonic acid metabolism-related genes in human esophageal squamous cell carcinoma. Int J Cancer 106 327-333, 2003

12. Boger PC, Shutt JD, Neale JR, Wilson SJ, Bateman AC, Holloway JW, Patel P and Sampson AP: Increased expression of the 5-lipoxygenase pathway and its cellular localization in Barrett's adenocarcinoma. Histopathology 61: 509-517, 2012.

13. Yang H, Jia X, Chen X, Yang CS and Li N: Time-selective chemoprevention of vitamin $\mathrm{E}$ and selenium on esophageal carcinogenesis in rats: the possible role of nuclear factor kappaB signaling pathway. Int J Cancer 131: 1517-1527, 2012.

14. Shi HY, Lv FJ, Zhu ST, Wang QG and Zhang ST: Dual inhibition of 5-LOX and COX-2 suppresses esophageal squamous cell carcinoma. Cancer Lett 309: 19-26, 2011
15. Amin MB, Edge S, Greene FL, et al: AJCC Cancer Staging Manual (M). 8th edition. New York, Springer, pp185-202, 2017.

16. Fang WK, Gu W, Li EM, Wu ZY, Shen ZY, Shen JH, Wu JY, Pan F, Lv Z, Xu XE, et al: Reduced membranous and ectopic cytoplasmic expression of DSC2 in esophageal squamous cell carcinoma: An independent prognostic factor. Hum Pathol 41: 1456-1465, 2010.

17. ShenZ,Cen S, Shen J, Cai W, Xu J,Teng Z, HuZ and Zeng Y: Study of immortalization and malignant transformation of human embryonic esophageal epithelial cells induced by HPV18 E6E7. J Cancer Res Clin Onco 126: 589-594, 2000.

18. Lisovyy OO, Dosenko VE, Nagibin VS, Tumanovska LV, Korol MO, Surova OV and Moibenko OO: Cardioprotective effect of 5-lipoxygenase gene (ALOX5) silencing in ischemia-reperfusion. Acta Biochima Pol 56: 687-694, 2009.

19. Ding X, Zhou X,Zhang H, Qing J, Qiang H and Zhou G: Triptolide augments the effects of 5-lipoxygenase RNA interference in suppressing pancreatic tumor growth in a xenograft mouse model. Cancer Chemother Pharmacol 69: 253-261, 2012.

20. Radmark O and Samuelsson B: 5-Lipoxygenase: Mechanisms of regulation. J Lipid Res 50 Suppl: S40-S45, 2009.

21. Nathoo N, Prayson RA, Bondar J, Vargo L, Arrigain S, Mascha EJ, Suh JH, Barnett GH and Golubic M: Increased expression of 5-lipoxygenase in high-grade astrocytomas. Neurosurgery 58: 347-354; discussion 347-354S, 2006

22. Tong WG, Ding XZ, Witt RC and Adrian TE: Lipoxygenase inhibitors attenuate growth of human pancreatic cancer xenografts and induce apoptosis through the mitochondrial pathway. Mol Cancer Ther 1: 929-935, 2002.

23. Gilbert B, Ahmad K, Roos J, Lehmann C, Chiba T, Ulrich-Rückert S, Smeenk L, van Heeringen S, Maier TJ, Groner B and Steinhilber D: 5-Lipoxygenase is a direct p53 target gene in humans. Biochim Biophys Acta 1849: 1003-1016, 2015.

24. Romano M, Catalano A, Nutini M, D'Urbano E, Crescenzi C, Claria J, Libner R, Davi G and Procopio A: 5-lipoxygenase regulates malignant mesothelial cell survival: Involvement of vascular endothelial growth factor. FASEB J 15: 2326-2336, 2001.

25. Ye YN, Wu WK, Shin VY and Cho CH: A mechanistic study of colon cancer growth promoted by cigarette smoke extract. Eur J Pharmacol 519: 52-57, 2005. 